\title{
The Relationship of Mean Perfusion Pressure (MPP) With Acute Kidney Injury (AKI) on Patients in Intensive Care Unit (ICU) at Haji Adam Malik General Hospital Medan
}

\author{
Sri Rahmadhona*, Asmin Lubis**, Dadik Wahyu Wijaya**, Akhyar Hamonangan Nasution** \\ *Resident of Anaesthesiology and Intensive Therapy, Faculty of Medicine, Universitas Sumatera Utara, Medan, Indonesia \\ **Departement of Anaesthesiology and Intensive Therapy, Faculty of Medicine, Universitas Sumatera Utara, Medan, Indonesia
}

Abstract

\section{$>$ Background:}

Acute Kidney Injury (AKI) is a common complication in patients with critical illness or multiple comorbid conditions who are hospitalized, especially at the Intensive Care Unit (ICU). AKI is associated with increased in short and long term mortality and morbidity. The connection between MAP and CVP on alteration of AKI causes researcher interested to investigate the correlation between Mean Perfusion Pressure (MPP) as a difference between MAP and CVP on the progression of $A K I$.

\section{$>$ Methods:}

Forty-two patients treated in the ICU who met inclusion criteria (ages 18-65 years and signed informed consent) and exclusion criteria (chronic kidney disease, refused to have Central Venous Catheters (CVC) installed, and heart disease). After CVC installation, we perform hemodynamic examination, MAP and CVP measurements, and serum creatinine examination after installation, 6 hours, 12 hours, and 48 hours after installation.

\section{$>$ Results:}

Significant differences were obtained during 12hour observation between MPP values in the AKI group with lower values than the non-AKI group $(p=0.009)$ and also obtained a significant difference during 48hours observation between MPP values in the AKI group with a lower value than the non-AKI group ( $p=0.001)$. MAP values showed a significant difference at 12-hour observation time with creatinine serum in the MAP group $<65 \mathrm{mmHg}$ higher than the MAP $\square$ 65mmHg group ( $p=0.035)$. MPP values showed significant differences 12-hours observation with creatinine serum in the MPP group $<55 \mathrm{mmHg}$ was higher than the MPP group $\square 55 \mathrm{mmHg}(\mathrm{p}=\mathbf{0 . 0 4 4})$. In addition, there was a correlation between creatinine serum increase with MPP decrease $(r=-0.476 ; p=0.001)$.

\section{$>$ Conclusion:}

Lower MPP tend to increase AKI occurence contrary higher MPP decrease AKI occurence at the observation. The mean MPP of AKI group was 90.6 ( $\square$ 24.2) $\mathrm{mmHg}$ at T0, $78.2(\square 22.6) \mathrm{mmHg}$ at T1, 67.2 ( $\square 20.9) \mathrm{mmHg}$ at $\mathrm{T} 3$, and $57,0(\square 20,2) \mathrm{mmHg}$ at $\mathrm{T} 4$ observation.

Keywords:- AKI, ICU, MAP, CVP, MPP, CVC.

\section{INTRODUCTION}

Acute Kidney Injury (AKI) is a complication commonly found in patients with critical illness or multiple comorbid conditions who are hospitalized, especially in Intensive Care Unit (ICU). AKI was associated with increased long and short-term mortality and morbidity. ${ }^{1,2}$

Hemodynamic regulation of renal blood flow and renal venous pressure is the key to determining renal function. Up until now, no technique have been found that can be used to assess and monitor intrarenal blood flow and renal perfusion pressure directly. It is thought that the mean perfusion pressure (MPP) might work as a surrogate. MPP is the difference between systemic mean arterial pressure and central venous pressure. ${ }^{3}$

Previous studies have found that lower MPP is associated with an increased risk of AKI but it is not yet known whether MPP also influences the risk of progression in patients with AKI that has already occurred. Study by Ostermann et al found that MPP over the 12 hour period following a diagnosis of AKI grade I was independently associated with the risk of progression to AKI III. There is a relationship between MPP and AKI progression especially in patients with ischemic heart disease or congestive heart disease, pre-existing hypertension, and those who have Mean Arterial Pressure / MAP <65 mmHg for 1 hour. Central venous pressure is a key component of MPP with an independent impact on the risk of progression to AKI III while MAP is not independently associated with progression. $^{2}$ 
According to study by Wong et al, it was said that when patients with chronic hypertension were analyzed separately with a large-scale randomized controlled study targeting MAP in cases of septic shock, it was found that high MAP improved kidney function and decreased the need for renal replacement therapy. But on the influence of MPP or Central Venous Pressure (CVP) there is no information on the premorbid value. Recent observational studies have found that patients with shock appear to experience AKI more often in conditions with large MPP deficits. Study by Wong et al found that patients with septic shock who had severe AKI had a greater MPP deficit during the first 24 hours in the ICU treatment room than patients who were otherwise. This difference is mainly determined by the excess of central venous pressure rather than the MAP deficit over the same time period and higher CVP value is significantly related to worsening AKI while other hemodynamic indices don't.. ${ }^{4}$

The relationship between MAP and CVP to worsen AKI causes researchers interested in examining the effect or correlation between MPP as a difference between MAP and CVP on the progression of AKI in patients treated in ICU at Haji Adam Malik General Hospital Medan.

\section{METHODS}

This study is an analytic study with a cohort prospective design carried out in the Intensive Care Unit at Haji Adam Malik General Hospital Medan. Consecutive sampling is a sample selection technique by which all subjects who come and meet the selection criteria are included in the study until the number of subjects is met. After obtaining approval from the Ethics Committee, Faculty of Medicine, University of North Sumatra, based on inclusion and exclusion criteria 42 research samples were collected.

The patient and family of the patient were explained during the visit regarding the research procedure for laboratory serum creatinine examination. In addition, we also informed the patient's family about the installation of CVC. Next, the researchers conducted a hemodynamic examination, MAP and CVP measurement, and blood drawn for serum creatinine examination (T0). At the $6^{\text {th }}$ (T1), $12^{\text {th }}(\mathrm{T} 2)$, and $48^{\text {th }}$ (T3) hours, hemodynamic examinations were re-evaluated, MAP and CVP measurements were taken, and blood samples were taken for serum creatinine examination. At last, the data would be tabulated, documented, and processed.

\section{RESULTS}

This study was attended by 42 subjects who met the inclusion criteria. The characteristics of this study were displayed based on gender, age, comorbid, urine production, use of vasopressor, MAP, MPP, and CVP.

\begin{tabular}{|c|c|c|c|}
\hline Characteristics & AKI & Non-AKI & P value \\
\hline \multicolumn{4}{|l|}{ Gender, n (\%) } \\
\hline Male & $8(19,04 \%)$ & $10(23,80 \%)$ & \multirow{2}{*}{0,738} \\
\hline Female & $9(21,42 \%)$ & $15(35,71 \%)$ & \\
\hline Age, Mean (SD) & $40,0( \pm 7,9)$ & $46,6( \pm 10,4)$ & $0.008 *$ \\
\hline \multicolumn{4}{|l|}{ Comorbid, n (\%) } \\
\hline $\mathrm{DM}$ & $5(12,8 \%)$ & $3(6,9 \%)$ & \\
\hline Hypertension & $2(5,1 \%)$ & $7(16,2 \%)$ & \\
\hline Pneumonia & $10(25,6 \%)$ & $12(27,9 \%)$ & 0,09 \\
\hline Sepsis & $12(30,7 \%)$ & $15(34,8 \%)$ & \\
\hline PPOK & $2(5,1 \%)$ & $1(2,3 \%)$ & \\
\hline Postoperative & $8(20,5 \%)$ & $5(11,6 \%)$ & \\
\hline Urine (ml/day), Mean (SD) & $2242( \pm 305)$ & $2081( \pm 260)$ & 0,088 \\
\hline Use of vasopressor, $\mathrm{n}(\%)$ & $8(47,0 \%)$ & $4(16 \%)$ & $0,002 *$ \\
\hline MAP, (mmHg), Mean (SD) & $98,3 \pm 23,6$ & $90,2 \pm 12,9$ & 0,215 \\
\hline MPP, (mmHg), Mean (SD) & $90,6 \pm 24,3$ & $82 \pm 12,8$ & 0,200 \\
\hline CVP, (mmHg), Mean (SD) & $8,02 \pm 1,4$ & $8,5 \pm 1,6$ & 0,380 \\
\hline
\end{tabular}

Note: Mann Whitney test (gender, comorbid, use of vasopressor), t-independent test (age, urine, MAP, MPP, CVP), *Significance if $\alpha<0,05$

Table 1:- Demographic Data Table 
Based on table 1 in this study, 42 people was found in which male and female genders were most commonly found in the non-AKI group, respectively $23.80 \%$ and $35.71 \%$. In addition, sepsis was found as the most common comorbid disease in this study. This study found that the characteristics of sex, comorbid disease, and the amount of urine were distributed evenly in both the AKI and non-AKI group, so that it was expected not to affect the MPP values and creatinine elevation that would be measured in this study.
This study also showed that the MAP, CVP, and MPP values were not significantly different at the beginning of the study which was the baseline value of the observation. However, the age and use of vasopressors differed in the two groups $(\mathrm{p}<0.05)$ so that it could be a biased factor in observing MPP values and creatinine elevations to be measured in this study.

\begin{tabular}{|c|c|c|c|c|c|}
\hline \multirow{2}{*}{ Characteristic } & \multicolumn{4}{|c|}{ MPP (mmHg) } & \multirow{2}{*}{ P value $^{\mathbf{b}}$} \\
\cline { 2 - 6 } & T0 & T1 & T2 & $0,001^{*}$ \\
\hline AKI & $90,6 \pm 24,2$ & $78,2 \pm 22,6$ & $67,2 \pm 20,9$ & $57,0 \pm 20,2$ & 0,70 \\
\hline Non-AKI & $82,0 \pm 12,8$ & $85,9 \pm 13,1$ & $89,4 \pm 14,1$ & $92,1 \pm 15,4$ & $0,001^{*}$ \\
\hline P value $^{\mathrm{a}}$ & 0,261 & 0,065 & $0,009 *$ & & T3 $^{*}$ \\
\hline
\end{tabular}

Note: $\mathrm{p}^{\mathrm{a}}$ : Mann Whitney test, $\mathrm{p}^{\mathrm{b}}$ : Friedman test. *Significance $\alpha<0,05$

Table 2:- The relationship between Mean Perfusion Pressure (MPP) and Acute Kidney Injury (AKI)

Based on table 2, it was found that the MPP value showed a significant difference in the T2 observation time (12 hour observation) and the MPP value in the AKI group was lower $(67.2 \pm 20.9 \mathrm{mmHg})$ compared to the non-AKI group (89.4 $\pm 14,1$ $\mathrm{mmHg}$ ). MPP values were also significantly different in T3 observations (48 hour observations) and MPP values in the AKI group were lower $(57.0 \pm 20.2 \mathrm{mmHg})$ compared to the non-AKI group $(92.1 \pm 15.4 \mathrm{mmHg})$. However, MPP values did not show significant differences in T1 observations (6 hour observations). These results indicate the MPP value in AKI tends to decrease starting from $\mathrm{T} 2$ observation (12 hour observation).

In addition, table 2 also shows that there was a significant decrease in MPP values in the AKI group with a range of decreases from $90.6 \pm 24.2 \mathrm{mmHg}$ at the beginning of the observation to $57.0 \pm 20.2 \mathrm{mmHg}$ at 48 hours of observation.

\begin{tabular}{|c|c|c|c|c|}
\hline \multirow{2}{*}{ MAP (mmHg) } & \multicolumn{4}{|c|}{ Creatinine serum (mg/dl) } \\
\cline { 2 - 5 } & T0 & T1 & T2 & $1,2 \pm 0,8$ \\
\hline$\geq 65$ & $1,2 \pm 1,0$ & $1,3 \pm 0,9$ & $2,0 \pm 1,4$ & $1,4 \pm 0,9$ \\
\hline$<65$ & $1,4 \pm 0,7$ & $1,9 \pm 1,3$ & $0,035^{*}$ & 0,052 \\
\hline P value & 0,362 & 0,080 & & 0,7 \\
\hline
\end{tabular}

Note: Mann Whitney test. *Significance $\alpha<0,05$

Table 3:- The relationship between Increased Creatinine Serum with Mean Arterial Pressure (MAP)

Based on table 3, it was found that the MAP value showed a significant difference at the time of T2 observation (12 hour observation) with serum creatinine values in the MAP group $<65 \mathrm{mmHg}$ higher $(2.0 \pm 1.4 \mathrm{mg} / \mathrm{dl})$ compared to the MAP group $\geq$ $65 \mathrm{mmHg}(1.2 \pm 0.8 \mathrm{mg} / \mathrm{dl})$. However, serum creatinine values did not show significant differences in T0 observations (initial creatinine examination), T1 (6 hour observations) and T3 (48 hour observations). These results indicate that serum creatinine in the MAP group $<65 \mathrm{mmHg}$ decreased significantly in 12-hour observations. The difference in serum creatinine was not significantly different in T3 observation (48 hour observation) due to treatment, although MAP was not independently associated with the progression of decreased kidney function.

\begin{tabular}{|c|c|c|c|c|}
\hline \multirow{2}{*}{ MPP } & \multicolumn{4}{|c|}{ Creatinine serum } \\
\cline { 2 - 5 } & T0 & T1 & $1,2 \pm 0,8$ & T3 \\
\hline$\geq 55$ & $1,2 \pm 1,0$ & $1,3 \pm 0,9$ & $2,1 \pm 1,4$ & $1,3 \pm 0,8$ \\
\hline$<55$ & $1,4 \pm 0,7$ & $1,9 \pm 1,3$ & $0,044 * 0,8$ \\
\hline P value & 0,362 & 0,080 & 0,068 \\
\hline
\end{tabular}

Note: Mann Whitney test. *Significance $\alpha<0,05$

Table 4:- The relationship between Increased Creatinine Serum with Mean Perfusion Pressure (MPP) 
Based on table 4, it was found that the MPP value showed a significant difference at the time of T2 observation (12 hour observation) with serum creatinine values in the MPP group $<55 \mathrm{mmHg}$ higher $(2.1 \pm 1.4 \mathrm{mg} / \mathrm{dl})$ compared to the MPP group $\geq$ $55 \mathrm{mmHg}(1.2 \pm 0.8 \mathrm{mg} / \mathrm{dl})$. However, serum creatinine values did not show significant differences in T0 observations (initial creatinine examination), T1 (6 hour observations) and T3 (48 hour observations). These results indicate serum creatinine in the MPP group $<55 \mathrm{mmHg}$ decreased significantly only at 12-hour observations.

\begin{tabular}{|c|c|c|c|}
\hline Variable & R & $\boldsymbol{P}$ \\
\hline Creatinine serum and MPP & $-0,476$ & 0,001 \\
\hline
\end{tabular}

Spearman's rank correlation, $* \mathrm{p}<0.05$

Table 5:- The correlation of Increased Creatinine Serum with Mean Perfusion Pressure (MPP)

\section{CONCLUSIONS}

There is a relationship between Mean Perfusion Pressure (MPP) with Acute Kidney Injury (AKI) in Intensive Care Unit (ICU) patients.

AKI sufferers are most common in women, with an average age of 40 years with the most comorbid diseases of sepsis and pneumonia.

The average MPP for AKI patients was $90.6( \pm 24.2)$ $\mathrm{mmHg}$ at $\mathrm{T} 0,78.2( \pm 22.6) \mathrm{mmHg}$ at $\mathrm{T} 1,67.2( \pm 20.9)$ $\mathrm{mmHg}$ at $\mathrm{T} 3$, and $57.0( \pm 20.2) \mathrm{mmHg}$ on $\mathrm{T} 4$ observation.

\section{REFERENCES}

[1]. Hoste E, Bagshaw S, Bellomo R, Cely CM, Coman R, Cruz DN, dkk. Epidemiology of Acute Kidney Injury In Critically Ill Patients: The Multinational AKI-EPI Study. Intensive Care Med. 2015;41(8):1411-23.

[2]. Ostermann, M., Hall, A., Crichton, S. Low Mean Perfusion Pressure Is A Risk Factor for Progression of Acute Kidney Injury in Critically Ill Patients-A Retrospective Analysis. BMC Nephrology 2017;18:151.

[3]. Firth J, Raine A, Ledingham J. Raised Venous Pressure: A Direct Cause of Renal Sodium Retention in Oedema? Lancet. 1988;1(8593):1033-5.

[4]. Wong, BT., Chan, MJ., Glassford, NJ., Martensson, J., Bion, V., Chai, SY., dkk. Mean Arterial Pressure and Mean Perfusion Pressure Deficit in Septic Acute Kidney Injury. Journal of Critical Care 2015. 